Francesco Moccia, Salvatore Tolone, Alfredo Allaria , Vincenzo Napolitano, D’Amico Rosa, Ferrante Ilaria, Manto Ottavia, Edoardo Cesaro, Ludovico Docimo, Landino Fei*

\title{
Lymph node ratio versus TNM system as prognostic factor in colorectal cancer staging. A single Center experience
}

https://doi.org/10.1515/med-2019-0058

received March 23, 2019; accepted May 21, 2019

\begin{abstract}
Objective: This study aims to establish the actual validity of the lymph node ratio (LNR) as a prognostic factor for colorectal cancer patients, and to verify differences of survival and disease-free interval. Methods: Patients referred with colorectal cancer who underwent potentially curative surgery between January 1997 and December 2011 were included. Lymph node ratio, TNM staging and survival were extracted from surgical, histological and follow-up records.
\end{abstract}

Results: Two hundred eigthy six patients with different stages of colorectal cancer underwent surgery, with comparison of survival prediction based on lymph node ratio and TNM staging. The overall survival rate was $78.3 \%$, the recurrence rate was $11.9 \%$ and the mortality rate was estimated as $21.7 \%$. Univariate analysis in relation to survival was significant for the following variables: serum level of CEA, CA 19.9 value, degree of histological differentiation, and tumor growth. There weren't any statistically significant differences for the LNR ( $\operatorname{LNR}</ \geq 0.16$ : $p=0.116$ ). The TNM system was effective both in discriminating between survival stages (Stage II vs. Stage III: $p=0.05$ ) and in differentiating sub-groups $(\mathrm{p}=0.05)$.

\footnotetext{
*Corresponding author: Landino Fei, Unit of Gastrointestinal Surgery, Department of Surgery, University of Campania “Luigi Vanvitelli”, Via Pansini 5, bld 17, ZIP 80100, Naples, Italy, Tel.+39 081566660 , Fax +39081566 6691 email: landino.fei@tin.it

Francesco Moccia, Alfredo Allaria, D’Amico Rosa, Ferrante Ilaria, Manto Ottavia, Edoardo Cesaro, Unit of Gastrointestinal Surgery, Department of Surgery, University of Campania “Luigi Vanvitelli", Naples, Italy

Salvatore Tolone, Ludovico Docimo, Unit of General, Mininvasive and Bariatric Surgery, Department of Surgery, University of Campania “Luigi Vanvitelli”, Naples, Italy Vincenzo Napolitano, Unit of Endoscopic Surgery, Department of Surgery, University of Campania “Luigi Vanvitelli”, Naples, Italy
}

Conclusions: LNR alone could not be considered a better prognostic factor than the TNM system. However, future studies are needed in a larger number of patients with a standardized surgical, pathological and medical protocol.

Keywords: Colorectal cancer; Lymph node Ratio; TNM staging

\section{Introduction}

Colorectal Cancer (CRC) is one of the most common cancers in Western countries and it is a frequent cause of death [1]. Among the parameters of the TNM system [2], there is a prevalence of lymph node status; in fact, the presence of metastasis in one or more lymph nodes determines the passage from one lower stage of disease to a higher stage, directly impacting on overall survival of patients. Presently, many researchers believe that patients suffering from CRC treated with radical surgery and with extensive lymphadenectomy have a better survival and greater disease-free interval [3-4]. Le Voyer et al. showed that an increased number of lymph nodes harvested is related to an increase of survival, both for patients with negative lymph nodes than for those with positive lymph nodes [5]. The number of metastatic lymph nodes harvested can depend on different features (i.e. the type and location of the tumor, the surgical technique and pathological examination) [6-7]. The AJCC in collaboration with The College of American Pathologists have defined the "minimum number" of 12 lymph nodes in order to define the lymphadenectomy as adequately performed [8]. It is believed that harvesting fewer than 12 lymph nodes can lead to the "stage migration" phenomenon. [9-11]. This is a bio-statistical model used to explain migration between stages with different prognoses, caused by inadequate lymph node sampling responsible for an erroneous sub-staging 
of the disease. In the seventh edition of TNM, the AJCC has proposed a further sub-division of the parameter $\mathrm{N}$ in N1a (only 1 metastatic node), N1b (2-3 positive nodes), N2a (4-6 positive lymph nodes) and N2b ( $\geq 7$ positive lymph nodes). This created new sub-groups that have the ability to further improve the prognostical assessment of individual patients. However, some still argue that the TNM system is lacking in stratifying risk subclasses of patients with lymph node involvement and that it does not take in account the total number of lymph nodes removed. Thus, on the basis of studies conducted on various human solid tumors [12-15], the use of the Lymph Node Ratio (LNR) has been proposed for CRC staging [5,16,17]. The LNR is defined as the ratio between the number of metastatic lymph nodes (LNs+) and total number of removed lymph nodes (total LNs) (LNs + / total LNs), and it is believed to have the potential to be a better prognostic factor than simple $\mathrm{N}$ assessment in the current TNM staging, whereas others $[18,19]$ do not recognize its validity.

We aimed to establish the actual validity of the LNR as a prognostic factor for CRC patients who underwent surgery with curative intent. Secondary endpoints were to verify possible differences, in terms of survival and disease-free interval, in groups of patients with fewer than 12 positive lymph nodes ( $-\mathrm{LN}_{\mathrm{s}}$ Group) in comparison to a group with more than 12 positive lymph nodes $\left(+\mathrm{LN}_{\mathrm{s}}\right.$ Group) (validity of the concept of "adequate lymphadenectomy").

\section{Materials and methods}

In a retrospective study, we enrolled 345 CRC patients who underwent potentially curative surgery (R0 resection) at our Unit of Gastrointestinal Surgery from January 1997 to December 2011. The R0 resection was defined as the removal of all macroscopic tumor, absence of microscopic residual tumor, negative resection margins and lymphadenectomy extended beyond the lymph nodes involved (with negative apical lymph nodes) [20]. Exclusion criteria were: patients with in situ cancer (stage 0), those in stage IV metastatic disease, those with unresectable locally advanced disease and / or peritoneal metastasis and/ or synchronous carcinomas. A further exclusion criteria was the presence of tumors of the rectum in EUS T3 $\mathrm{N}+$ stage, in which patients were candidates for neoadjuvant therapy, because of the alterations caused by preoperative radiochemotherapy on the lymph node structure and on prognosis [21,22]. Also, we excluded patients who fulfilled Amsterdam II criteria for colorectal cancer syndrome, hereditary non-polyposis colorectal cancer (HNPCC) or carcinoma associated with chronic Inflammatory Bowel Disease (IBD). All patients, at the time of informed consent, signed an authorization form for the processing of personal data. The study was approved by the local Institutional Review Board. The anatomical specimens were fixed by immersion in formalin immediately after surgical excision, with the ratio of 1:10 to 1:20 of specimen to volume and amount of fixative liquid for a period of no less than 24-48 hours depending on the specimen dimensions. Lymphovascular and / or perineural infiltration, although present in some samples, has not been evaluated systematically by pathologists; because of this, while recognizing its prognostic value [22,23], we decided not to mention it as an additional characteristic. With regard to patients with stage II and III (Dukes's stage B and C), the LNR was calculated by dividing the number of metastatic lymph nodes by the total number of excised lymph nodes $\left(\mathrm{LN}_{\mathrm{s}}+/\right.$ total $\mathrm{LN}_{\mathrm{s}}$ ); by doing so, we evaluated the population with positive lymph nodes, also in reference to the critical value or threshold value of the LNR calculated using statistical methods $(</ \geq 0.16)$.

After discharge, all patients were followed up by the team of oncologists to continue treatment with adjuvant chemotherapy or with exclusive follow-up; they were monitored with updates on their clinical history, physical examination, determination of carcinoembryonic antigen (CEA) levels in serum and CA 19.9 values (every 3 months for the first 2 years, then every 6 months for a total 5 years). Colonoscopies were performed after one year and then every three years; liver ultrasounds were performed every 6 months for 3 years and then in the 4th and 5th year. CT scans of chest, abdomen and pelvis were conducted annually for 5 years. We concluded the period of follow-up and data collection in January 2014, with a mean 5-year follow-up for each patient.

For data collection, a dedicated Microsoft Works database (version 7.0) was used. We recorded the following clinic-pathological parameters: age, gender, tumor type, size, location in the colon, serum level of CEA (normal value 3,5ng/ml) and CA 19.9 (normal value $<37 \mathrm{U} / \mathrm{ml}$ ), pathologic stage (pTNM), and degree of histological differentiation (well, moderately or poorly differentiated). Moreover we calculated the number of resected lymph nodes and the number of metastatic lymph nodes, the LNR in subjects $\mathrm{N}+$, postoperative complications and the evolution of the disease, as the absence or presence of recurrent disease, death and cause of death. Patients who died from causes other than colorectal cancer with no evidence of disease and patients lost to follow-up were 
considered as censored in reference to the death rate from cancer or disease-specific survival.

\section{Statistical analysis}

The statistical population analysis was performed using the SPSS software version 17.0 for Windows. The 5-year survival was calculated according to the Kaplan-Meier method; Log-rank test was used to determine statistically significant differences in the examined groups. Multivariate analysis was performed with the Cox model. Statistical analysis was defined as significant when $\mathrm{p}$ values $<0.05$. The critical value of the LNR $(</ \geq 0.16)$ was calculated with statistical method, dividing the population into quartiles of patients with positive lymph nodes (stage III - TNM System) [24, 25].

\section{Results}

From the initial cohort of 345 CRC subjects, we excluded patients with stage I due to small sample size (only 12 cases). Eight patients died from postoperative complications that occurred in the first thirty days after surgery and were also excluded. The final study population was constituted as follows: 286 patients, 166 (58.1\%) males, $120(41.9 \%)$ females, mean age $65 \pm 10$ years (range 28-92, median 65; no statistical difference was observed regarding age in the two sexes: $\mathrm{p}=0.751$ ). According to the TNM system, 15 patients were classified as stage I (Dukes's stage A), 156 patients as stage II (Dukes's stage B) without lymph node involvement and 115 patients as stage III (Dukes's stage C) with metastatic lymph nodes, respectively. Among the study population, we identified $104(36.5 \%)$ patients who received inadequate lymphadenectomy (<12 lymph nodes removed (- $\mathrm{LN}_{\mathrm{s}}$ Group)) and another 182 (63.5\%) subjects who received an adequate lymphadenectomy $\left(\geq 12\right.$ lymph nodes removed $\left(+\mathrm{LN}_{\mathrm{s}}\right.$ Group)).

Table 1 depicts the principal epidemiological characteristics and clinical-pathological features of the cohort. The overall survival rate was $78.3 \%$, the recurrence rate was $11.9 \%$ and the mortality rate was estimated as $21.7 \%$. The analysis of survival curves showed that the disease relapse occurred more frequently during the first 18-24 months after diagnosis.

Of the total removed lymph nodes (average $14 \pm 6.933$, range 2-46), 388 were metastatic nodes (mean $1.36 \pm 3.664$, range $1-18)$. In patients treated before the year 2000, the isolated lymph nodes number (mean $13.5 \pm 6.585$, range 2-28) was lower compared to the lymph nodes retrieved in patients operated on after the year 2000 (mean $15 \pm$ 6.933, range 2- 46), $\mathrm{p}<0.05$. Patients with harvested lymph nodes less than 12 showed a significantly lower metastatic lymph nodes number compared to patients with lymph nodes $\geq 12,37 \%$ and $63 \%$ ( $\mathrm{p}<0.001)$, respectively; in stage III patients, the range of the LNR was between 0.03 and 1 , the mean LNR was $0.26 \pm 0.25$; the median LNR was 0.17 . The LNR variable distributed in stages (IIIA, IIIB and IIIC) are shown in Figure 1.

Univariate analysis of clinical and pathological features in relation to survival was significant for the following variables (Tab. 1): serum level of CEA ( $p=0.001)$, CA $19.9(p=0.004)$, degree of histological differentiation ( $p<0.017)$, tumor growth $(p=0.016)$, lymph node status $(\mathrm{p}=0.18)$, and pathological TNM staging $(\mathrm{p}=0.14)$ (Fig. 2a-c). There wasn't any statistically significant difference for the LNR (LNR $</ \geq 0,16: p=0.116)$. The TNM system was effective both in discriminating between survival stages (Stage II vs. Stage III ( $p=0.05)$ (Fig. 4a-b) and in differentiating sub-groups $(\mathrm{p}=0.05)$. The survival curves in $-\mathrm{LN}_{\mathrm{s}}$ and $+\mathrm{LN}_{\mathrm{s}}$ groups were $75 \%$ and $80.2 \%$, respectively, with a highly significant increase in the relative risk of recurrence in the group $-\mathrm{LN}_{\mathrm{s}}$ versus group $+\mathrm{LN}_{\mathrm{s}}(21,15 \%$ vs $6.6 \%$; p <0.001) (Tab. 2 - 3 and Fig. 5). In addition, patients in the $-\mathrm{LN}_{\mathrm{s}}$ group showed a significantly lower percentage of lymph node metastases compared to $+\mathrm{LN}_{\mathrm{s}}$ group $(37.5 \%$ vs $41.7 \%$; $\mathrm{p}<0.001)$.

\section{Discussion}

Surgery with curative intent, with or without chemotherapy and radiotherapy, is currently the treatment of choice for CRC; its staging is referred to the TNM System. The present study shows that an LNR $>0.16$ wasn't an independent risk factor in predicting overall survival and disease-free interval, whereas the seventh TNM System edition seemed to be the most effective prognostic tool to predict disease-free interval and overall survival in CRC patients, even in differentiating sub-groups. The presence of more positivelymph nodes ( $+\mathrm{LN}_{\mathrm{s}}$ group) was related to an increased relative risk of disease recurrence, even if it was correlated to an increased presence of metastatic nodes. As well as for other digestive cancers [26-29], the use of the LNR was proposed for CRC, assuming it as a possibly better independent prognostic factor for the $\mathrm{N}$ parameter than TNM staging $[5,16,17]$. However, concern exists about the real role of the LNR in CRC. Berger et al. [30] investigated 
Table 1: Clinico-pathological features and univariate analysis of overall survival in colorectal cancer patients who underwent potentially curative surgery.

\begin{tabular}{|c|c|c|c|c|}
\hline & Patients N. (\%) & Death events $\mathrm{N}$ & Survival (\%) & p-value \\
\hline & 286 & 62 & 0.783 & - \\
\hline \multicolumn{5}{|l|}{ Age } \\
\hline$<65$ years & $141(49)$ & 29 & 75.8 & 0.57 \\
\hline$>65$ years & $145(50.7)$ & 37 & 65.4 & \\
\hline \multicolumn{5}{|l|}{ Sex } \\
\hline Female & $120(41.4)$ & 23 & 76.29 & 0.486 \\
\hline Male & $166(58.1)$ & 39 & 69.29 & \\
\hline \multicolumn{5}{|l|}{ CEA } \\
\hline$<3.5 \mathrm{ng} / \mathrm{ml}$ & $146(51.4)$ & 17 & 86.82 & 0.001 \\
\hline$>3.5 \mathrm{ng} / \mathrm{ml}$ & $140(48.6)$ & 45 & 52.63 & \\
\hline \multicolumn{5}{|l|}{ CA 19.9} \\
\hline$<37 \mathrm{U} / \mathrm{ml}$ & $238(83.2)$ & 46 & 76.04 & 0.004 \\
\hline$>37 \mathrm{U} / \mathrm{ml}$ & $48(16.8)$ & 16 & 50 & \\
\hline \multicolumn{5}{|l|}{ Tumor site } \\
\hline Right Colon & $75(26.2)$ & 16 & 72.88 & 0.027 \\
\hline Transverse Colon & $8(2.8)$ & 4 & 0 & \\
\hline Left Colon & $119(41.6)$ & 21 & 78.57 & \\
\hline Rectum & $84(29.4)$ & 21 & 66.57 & \\
\hline \multicolumn{5}{|l|}{ Tumor Size } \\
\hline$<3 \mathrm{~cm}$ & $60(20.1)$ & 14 & 69.57 & 0.145 \\
\hline$>3 \mathrm{~cm}$ & $226(79.9)$ & 48 & 80 & \\
\hline \multicolumn{5}{|l|}{ Histologic Grading } \\
\hline G1 & $135(47.2)$ & 20 & 82 & 0.017 \\
\hline G2 & $121(42.3)$ & 30 & 67.03 & \\
\hline G3 & $30(10.5)$ & 12 & 33.33 & \\
\hline \multicolumn{5}{|l|}{ pT } \\
\hline 1 & $9(3.2)$ & 0 & 100 & 0.161 \\
\hline 2 & $24(8.4)$ & 3 & 85.71 & \\
\hline 3 & $202(70.6)$ & 42 & 73.75 & \\
\hline 4 & $51(17.8)$ & 17 & 50 & \\
\hline \multicolumn{5}{|l|}{$\mathrm{pN}$} \\
\hline 0 & $171(59.8)$ & 31 & 77.86 & 0.184 \\
\hline 1 & $75(26.2)$ & 20 & 63.64 & \\
\hline 2 & $40(14)$ & 11 & 62.07 & \\
\hline \multicolumn{5}{|l|}{ pTNM Stage } \\
\hline 1 & $9(3.2)$ & 0 & 100 & 0.161 \\
\hline IIA & $125(43.7)$ & 21 & 79.81 & \\
\hline IIB & $26(9.1)$ & 10 & 37.5 & \\
\hline IIC & $4(1.4)$ & 0 & 100 & \\
\hline IIIA & $19(6.7)$ & 3 & 81.25 & \\
\hline IIIB & $69(24.1)$ & 20 & 59.18 & \\
\hline IIIC & $26(9.1)$ & 8 & 55.56 & \\
\hline \multicolumn{5}{|l|}{ LNR } \\
\hline$<0.16$ & $227(79.4)$ & 45 & 72.27 & 0.116 \\
\hline$>0.16$ & $59(20.26)$ & 17 & 59.52 & \\
\hline \multicolumn{5}{|l|}{ Relapse } \\
\hline Yes & 34 (11.9) & 0 & 67.7 & 0.001 \\
\hline No & $252(88.1)$ & 62 & 100 & \\
\hline
\end{tabular}

LNR=Lymph node Ratio 


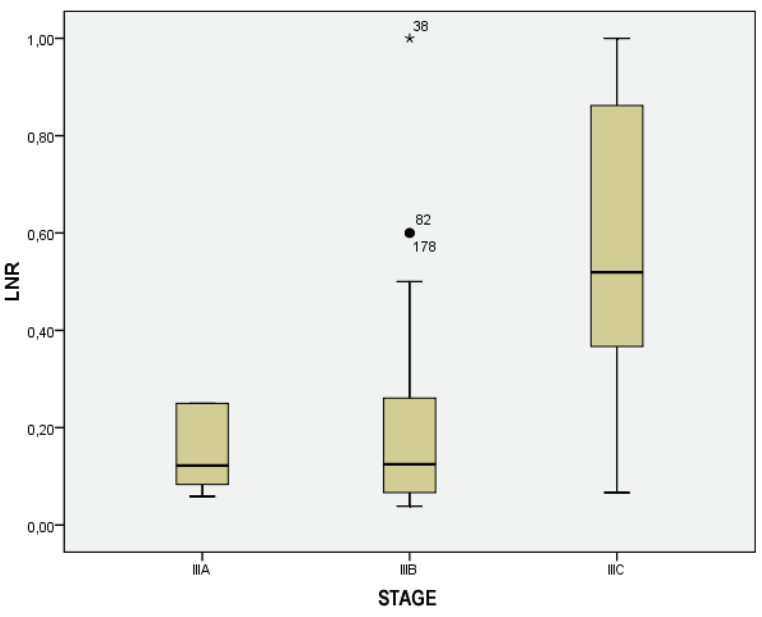

Figure 1: Box-plot diagram showing lymph node ratio (LNR) values in colorectal cancer Stages IIIA, IIIB and IIIC.

b)

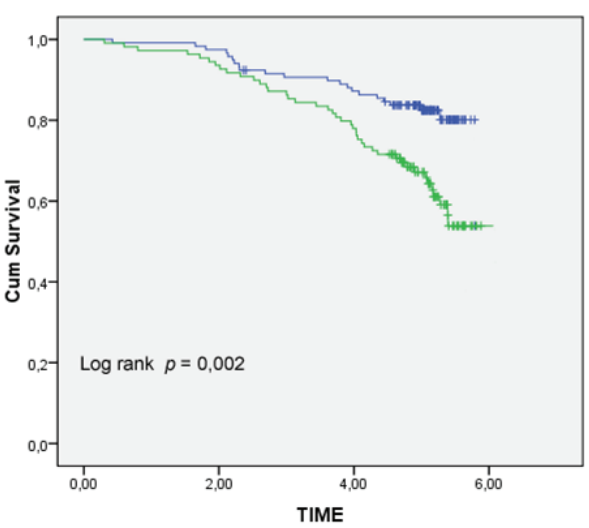

Figure 2b: Overall survival (OS) according to circulating CEA (< $3.5 \mathrm{ng} / \mathrm{ml}$ vs $\geq 3.5 \mathrm{ng} / \mathrm{ml}, \mathrm{p}=0.001$

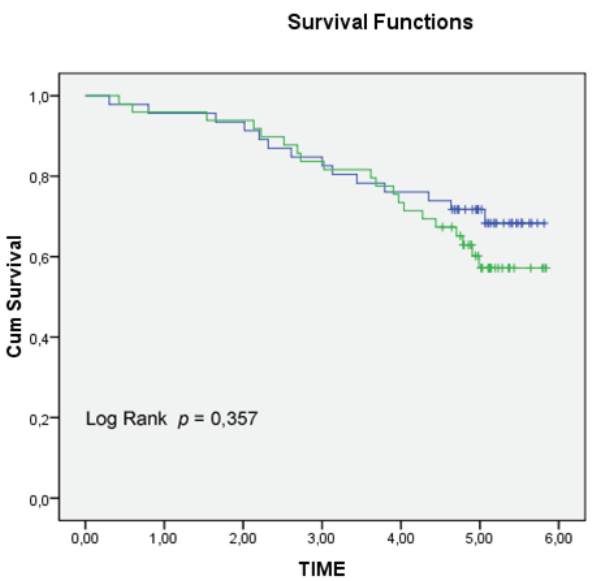

Figure 3: Overall survival (OS) according to lymph node ratio (LNR) $(<0.16$ vs $\geq 0.16, p=0.116)$.

$\neg 1<0,16$
$\neg 2>0,16$
+1 -censored

+ 2 -censsored a) Survival Functions

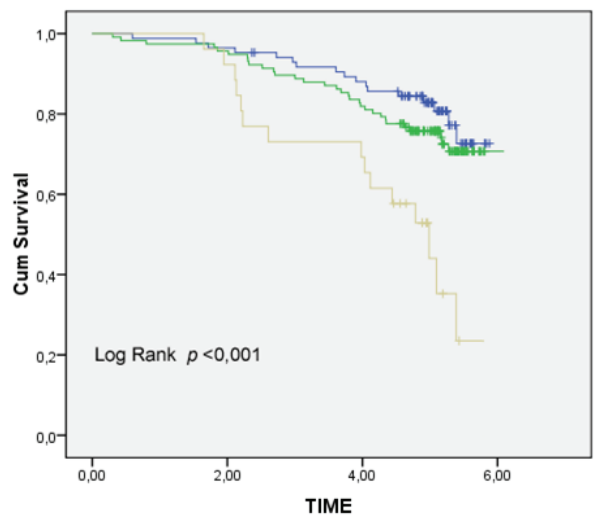

GRADING

$\neg 1$
$\neg 2$
$\neg 3$

+1 -censored

-2-censored

Figure 2a: Overall Survival (OS) according to histological grading (G1 and $G 2$ vs. $G 3, p=0.017)$.

c)

Survival Functions

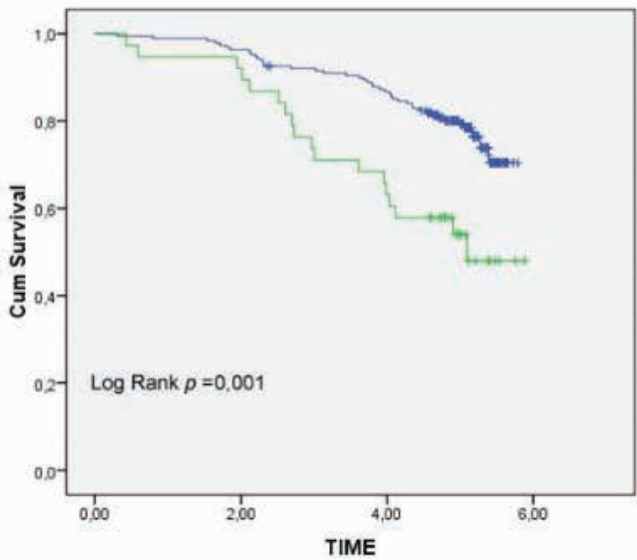

CA19_9

$\rightarrow 1$ +1 -censored
+2 -censored

Figure 2c: Overall survival (OS) according to circulating CA 19.9 (< $37 \mathrm{U} / \mathrm{ml}$ vs $\geq 37 \mathrm{U} / \mathrm{ml}, \mathrm{p}=0.004)$.

a)

Survival Functions

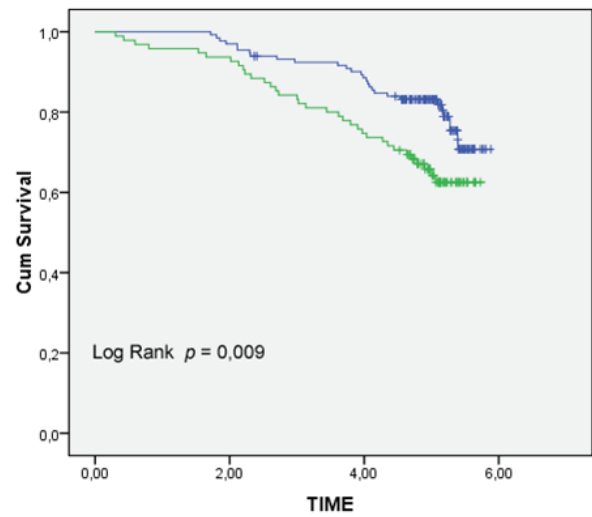

STAGE II - III

$\neg$ III
+2-censored

+2 -censored
+3 -censored

Figure 4a: Overall Survival (OS) in colorectal cancer patients Stage II and III ( $p<0.05)$; 


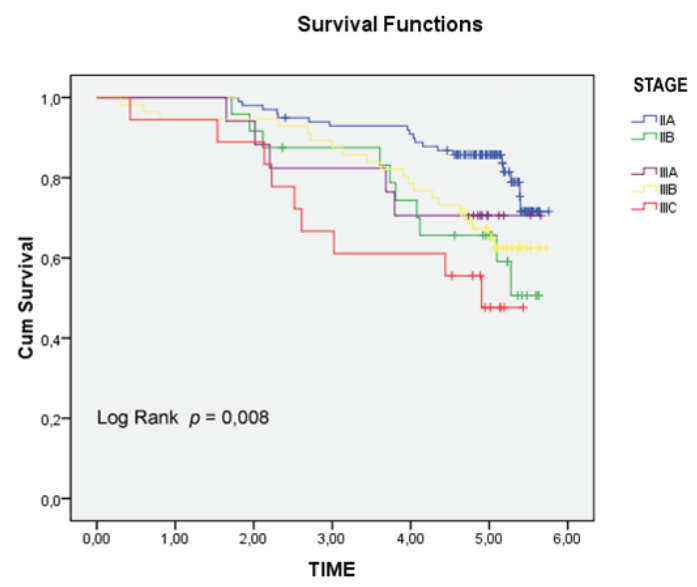

Figure 4b: Overall Survival (OS) in different subgroups of Stage II and III $(p=0.05)$.

the relationship between the LNR and survival in patients with stage II and III of CRC with an average of 13 harvested lymph nodes. The LNR was an important prognostic factor for overall survival and disease-free interval, especially in patients with 10 to 15 lymph nodes removed, greater than or equal to 15 , than in those with less than 10 lymph nodes removed. Lee et al. [31] assessed the prognostic significance (disease-free survival) of the LNR, in patients with stage III CRC. They showed that the value of the LNR increases proportionally with the increased number of metastatic lymph nodes and to be closely related to the surgical intervention quality. Subsequently, Wang et al. [19] suggested that the LNR can overcome the influence of the number of positive lymph nodes, especially in patients with CRC stage III B and III C; these latter are most likely to be understaged or overstaged, unless a correction parameter is included to possibly make a sub-classification of the stage. According to the variable $\mathrm{N}$, both have the same prognosis if all other conditions are equal. However, the survival in the first case is worse than in the second, and the LNR can distinguish between the two situations: the LNR is equal to 1 in the first situation and to 0.05 in the second situation. It is known that the number of lymph nodes harvested and the number of positive lymph nodes depend to a large extent on features related to the patient, the biology of the tumor, the host's immune response, the surgeon's technique, the anatomical factor, the pathologist's experience and the ability to find the positive nodes.

This implies that the number of $\mathrm{LN}_{\mathrm{s}}$ collected and the number of positive nodes $\left(\mathrm{LN}_{\mathrm{s}}+\right)$ are not very reliable and do not represent solid prognostic indicators. The use of the LNR was advocated to be able to avoid the dependence on such factors as the probability that a positive $\mathrm{LN}+$ is not dependent on the number of $\mathrm{LN}_{\mathrm{s}}$ collected. The impor-

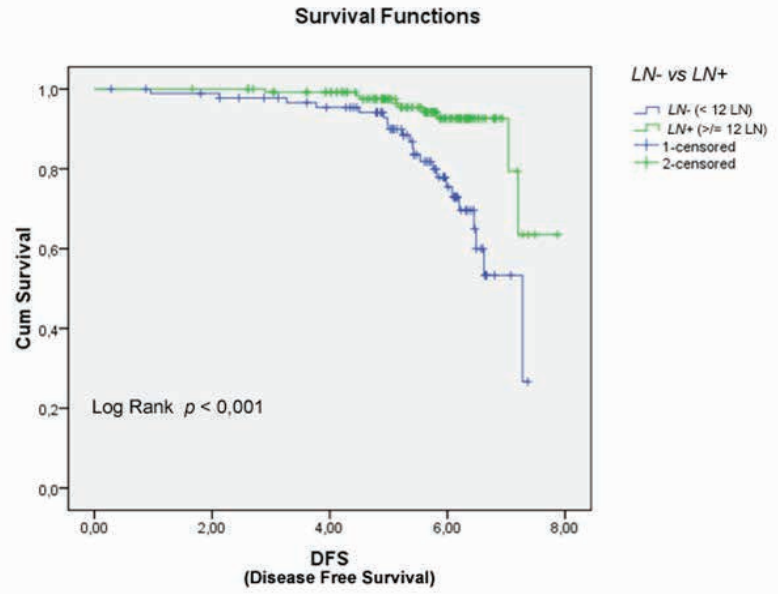

Figure 5: Disease-free survival (DFS) in colorectal cancer patients with lymph node negative (LNs-) and lymph node positive (LNs+) (relapse rate $6.6 \%$ vs $21.15 \%$, respectively; $p<0.001$ ).

tance of the role of the surgeon in performing adequate dissection and the pathologist's ability to identify and collect enough $\mathrm{LN}_{\mathrm{s}}$ should be emphasized. Wang et al. [19] proposed to incorporate the LNR in the current staging of colorectal carcinoma in stage III. Also, the LNR was found to be an independent prognostic factor better than pN-stage, pT-stage, pM- internships, staging according to the UICC and the grade of the tumor. To determine the impact of the LNR within the AJCC classification system, Lee et al [31] analyzed the 3-year disease free survival of patients in stage IIIA, IIIB and IIIC in relation to the LNR. They also looked at the impact of the LNR compared with the number of lymph nodes removed. AThe authors stated that patients with stage III cancer who underwent curative resection may be included in a new classification that unites the TNM system and the LNR. In this new staging, the influence of LNR prognosis is proportional to the number of lymph nodes removed. However, despite conflicting opinions of different researchers about the LNR, what unites the opposing factions are the numerous criticisms directed mainly at the patient selection criteria, the threshold values used, the heterogeneity of the data collected, an the different methods of study responsible for contrasting results [21, 32-35]. In the study by Schiffmann et al. [18] the prognostic power of the LNR was compared to the pN parameter of TNM system; $\mathrm{pN}$ parameter was a stronger feature in predicting overall survival and disease-free survival than the LNR. In a recent study conducted in 1,127 patients, Zhang J. et al. [25] showed that LNR was independent of the total number of lymph nodes retrieved; however, the authors demonstrated through a linear regression analysis that 
Table 2: Disease Free Survival between -LNs group and + LNs group (relapse rate: $21.15 \%$ vs $6.6 \%$, respectively; $p<0.001$ ).

\begin{tabular}{|c|c|c|c|c|}
\hline \multicolumn{5}{|c|}{ Case Processing Summary } \\
\hline \multicolumn{3}{|l|}{$-/+$ LNs groups } & \multicolumn{2}{|l|}{ Censored } \\
\hline & Total N & Events N. (\%) & N. & Percent \\
\hline$-\mathrm{LNs}$ & 104 & $22(21.15)$ & 82 & 78.8 \\
\hline$+\mathrm{LNs}$ & 182 & $12(6.6)$ & 170 & 93.4 \\
\hline Overall & 286 & $34(11.88)$ & 252 & 88.1 \\
\hline \multicolumn{5}{|c|}{ Overall Comparison } \\
\hline Log Rank & Chi-Square & $\mathrm{df}$ & & significance \\
\hline (Mantel-Cox) & 14.157 & 1 & & $<0.001$ \\
\hline
\end{tabular}

Test of equality of survival distribuitions for the different levels of $+\mathrm{LN} /-\mathrm{LN}$

-LNs group $=<12$ lymph nodes removed

+ LNs group $=\geq 12$ lymph nodes removed

Table 3: Disease Free Survival between -LN group and +LN (survival rate: $75 \%$ vs $80.2 \%$, respectively; $p<.002$ ).

\begin{tabular}{|c|c|c|c|c|}
\hline \multicolumn{5}{|c|}{ Case Processing Summary } \\
\hline \multicolumn{3}{|l|}{$-/+\mathrm{LN}_{\mathrm{s}}$ groups } & \multicolumn{2}{|l|}{ Censored } \\
\hline & Total $\mathrm{N}$ & Events N. (\%) & N. & Percent \\
\hline$-\mathrm{LN}_{\mathrm{s}}$ & 104 & $26(25)$ & 78 & 75 \\
\hline$+\mathrm{LN}_{\mathrm{s}}$ & 182 & $36(19.78)$ & 146 & 80.2 \\
\hline Overall & 286 & $62(21.7)$ & 224 & 78.3 \\
\hline \multicolumn{5}{|c|}{ Overall Comparison } \\
\hline Log Rank & Chi-Square & $\mathrm{df}$ & & significance \\
\hline (Mantel-Cox) & 14.157 & 1 & & $<0.002$ \\
\hline
\end{tabular}

Test of equality of survival distribuitions for the different levels of $+\mathrm{LN} /-\mathrm{LN}$

-LNs group $=<12$ lymph nodes removed

+ LNs group $=\geq 12$ lymph nodes removed

the number of metastatic lymph nodes is directly proportional to the number of lymph nodes removed. This supports the hypothesis that if the lymphadenectomy is inadequate, the risk of leaving lymph node metastases is real. Thus, the LNR seems to be a reliable parameter, far more than the number of positive lymph nodes, and could be used as an independent prognostic factor in CRC patients who undergo radical resection of the tumor with positive lymph node metastases and with an adequate lymphadenectomy. Currently, referring to the AJCC - ACP guidelines, the minimum number to obtain an adequate lymphadenectomy must be equal to or greater than 12 lymph nodes. In support of this study, Lykke et al [36] showed that patients that have more than 12 lymph nodes harvested are more likely to be identified in stage III than in stage II according to the phenomenon of "stage migration". Therefore, it is hypothesized that in patients who have less than 12 lymph nodes isolated that there might be an error in the staging of the disease (understaging) for any unrecognized lymph node micro or macro metastases. To avoid the phenomenon of stage migration, AJCC guidelines also recommend adjuvant chemotherapy for patients with negative lymph nodes in which less than 12 lymph nodes were isolated, because this population is considered at high risk of relapse [37,38]. This provision, in addition to limiting the error in the staging and management of cancer patients, restricts the use of the LNR, proposed initially just to minimize the effects of the phenomenon stage migration [39].

In the present study, we showed that until year 2000, the number of harvested lymph nodes was lower compared to the lymph nodes retrieved in patients operated after year 2000; recent guidelines have sensitized the surgeons and pathologists to seek the highest number of lymph nodes as possible. Furthermore, according to our experience and to several studies $[26,28,33]$, increasing the number of lymph nodes removed significantly reduces relative risk of relapse $(\mathrm{p}<0.001)$ and increases the possibility of isolating metastatic lymph nodes, otherwise unrecognized. For this reason, we fully share the AJCC decision to treat "as patients with lymph node" metastases, all patients with pNo less than twelve lymph nodes 
removed, in order to stem the statistical paradox of stage migration, independently of LNR results. Finally, we found that already known risk factors such as histological grade and the level of tumor markers (CEA and CA 19.9) have valid prognostic value.

Despite the very tight selection criteria, the present study has some limitations: it is a purely retrospective observational study, and the results may be affected by certain factors such as the adjuvant chemotherapy treatment.

\section{Conclusions}

In the present study, we showed that the LNR alone could not be considered a better prognostic factor than the TNM system. Also, the TNM system, considered in its entirety (ie Tumor, Node and Metastasis) is a reliable parameter, reproducible and with great prognostic value in patients with CRC. However, future studies are needed in a large number of CRC patients with a standardized surgical, pathological and medical protocol to verify the potential prognostic role of combined or separated risk factors.

Sources: No grant support or financial relationship were provided for this study

Authors contribution: FM, ST, AA, VN, MO, FI, DAR, LD and LF contributed to conception of the work, analysis, drafting the work, final approval of the manuscript, and agreement to be accountable for all the aspects of the work

Conflict of interest: The authors declare that they have no conflicts of interest

\section{Reference}

[1] Jemal A, Bray F, Melissa M et al. Global Cancer statistics 2011. CA Cancer J Clin 2011; 61: 69-90

[2] Edge SB, Byrd DR, Compton CC et al. AJCC cancer staging manual, 7th edn. Springer 2010, New York

[3] Johnson PM, Porter GA, Ricciardi R et al. Increasing negative lymph node count is independently associated with improved long-term survival in stage IIIB and IIIC colon cancer. J Clin Oncol 2006; 24: 3570-3575

[4] Chang GJ, Rodriguez-Bigas MA, Skibber JM et al. Lymph node evaluation and survival after curative resection of colon cancer: systematic review. J Natl Cancer Inst 2007; 99: 433-441

[5] Le Voyer TE, Sigurdson ER, Hanlon AL et al. Colon cancer survival is associated with increasing number of lymph nodes analyzed: a secondary survey of intergroup trial INT-0089. J Clin Oncol 2003; 21: 2912-2919

[6] Wong SL, Ji H, Hollenbeck BK, Morris AM et al. Hospital lymph node examination rates and survival after resection for colon cancer. JAMA 2007; 298: 2149-2154

[7] Evans MD, Barton K, Rees A et al. The impact of surgeon and pathologist on lymph node retrieval in colorectal cancer and its impact on survival for patients with Dukes' stage B disease. Colorectal Dis 2008; 10: 157-164

[8] Compton CC, Fielding LP, Burgardt LJ et al. Prognostic factors in colorectal cancer. College of American pathologists consensus statement 1999. Arch Pathol Lab Med 2000; 124: 979994

[9] Namm J, Melody Ng, Chowdhury SR et al. Quantitating the impact of stage migration on staging accuracy in colorectal cancer. J Am Coll Surg 2008; 207: 882-887

[10] George S, Primrose J, Talbot R et al. Will Rogers revisited: prospective observational study of survival of 3592 patients with colorectal cancer according to number of nodes examined by pathologists. Brit J Cancer 2006; 95:841-847

[11] Feinstein AR, Sosin DM, Wells CK. The Will Rogers phenomenon. Stage migration and new diagnostic techniques as a source of misleading statistics for survival in cancer. N Engl J Med 1985; 312: 1604-1608

[12] Sianesi M, Bezer L, Del Rio P et al. The Node Ratio as Prognostic Factor after Curative Resection for Gastric Cancer. J Gastrointest Surg 2010; 14: 614-619

[13] Hsu WH, Hsu PK, Hsieh CC et al. The metastatic lymph node number and ratio are indipendent prognostic factors in esophageal cancer. J Gastrointest Surg 2009; 13: 1913-1920

[14] Vinh-Hung V, Verkooijen HM, Fioretta G et al. Lymph node ratio as an alternative to $\mathrm{pN}$ staging in node-positive breast cancer. J Clin Oncol 2009; 27: 1062-1068

[15] Riediger $\mathrm{H}$, Keck $\mathrm{T}$, Wellner $\mathrm{U}$ et al. The lymph node ratio is the strongest prognostic factor after resection of pancreatic cancer. J Gastrointest Surg 2009; 13(7): 1337-1344

[16] Baxter NN, Virnig DJ, Rothenberger DA et al. Lymph node evaluation in colorectal cancer patients: a population-based study. J Natl Cancer Inst 2005; 97: 219-225

[17] Vather R, Sammour T, Kahokehr A et al. Lymph node evaluation and long-term survival in stage II and stage III colon cancer: a national study. Ann Surg Oncol 2009; 16: 585-593

[18] Schiffmann L, Eiken AK, Gock M, Klar E. Is the lymph node ratio superior to the Union for International Cancer Control (UICC) TNM system in prognosis of colon cancer? World J Surg Oncol 2013; 11:79

[19] Wang LP, Wang HY, Cao R, Zhu C, Wu XZ. Proposal of a new classification for stage III colorectal cancer based on the number and ratio of metastatic lymph nodes. World J Surg 2013; 37(5): 1094-102

[20] Chapuis PH, Dent OF, Bokey EL et al. Adverse histopathological findings as a guide to patient management after curative resection of node positive colon cancer. Br J Surg 2004; 91: 349354.

[21] Peschaud $\mathrm{F}$, Benoist $\mathrm{S}$, Julié $\mathrm{C}$ et al. The ratio of metastatic to examined lymph nodes is a powerful independent prognostic factor in rectal cancer. Ann Surg 2008; 248:1067-1073

[22] Chandrasinghe PC, Ediriweera DS, Hewavisenthi J et al. The total number of lymph nodes harvested is associated with 
better survival in stages II and III colorectal cancer. Indian J Gastroenterol 2013. [Epub ahead of print] PubMed PMID: 24048680

[23] Lu YJ, Lin PC, Lin CC et al. The impact of the lymph node ratio is greater than traditional lymph node status in stage III colorectal cancer patients. World J Surg 2013; 37(8): 1927-1933

[24] Rausei S, lovino D, Tenconi S et al. Impact of lymph node ratio on survival of colorectal cancer patients. Int I Surg 2013; 11 Suppl 1: S95-9

[25] Zhang J, Lv L, Ye Y et al. Comparison of metastatic lymph node ratio staging system with the 7th AJCC system for colorectal cancer. J Cancer Res Clin Oncol 2013; 139(11): 1947-1953

[26] Truong PT, Vinh-Hung V, Cserni G et al. The number of positive nodes and the ratio of positive to excised nodes are significant predictors of survival in women with micrometastatic nodepositive breast cancer. Eur J Cancer 2008; 44: 1670-1677

[27] Slidell MB, Chang DC, Cameron JL et al. Impact of total lymph node count and lymph node ratio on staging and survival after pancreatectomy for pancreatic adenocarcinoma: a large, population-based analysis. Ann Surg Oncol 2008; 15: 165-174

[28] Mariette C, Piessen G, Briez $\mathrm{N}$ et al. The number of metastatic lymph nodes and the ratio between metastatic and examined lymph nodes are independent prognostic factors in esophageal cancer regardless of neoadjuvant chemoradiation or lymphadenectomy extent. Ann Surg 2008; 247: 365-371

[29] Marchet A, Mocellin S, Ambrosi A et al. The prognostic value of $\mathrm{N}$-ratio in patients with gastric cancer. Validation in a large, multicenter series. Eur J Surg Oncol 2008; 34: 159-165

[30] Berger AC, Sigurdson ER, LeVoyer T et al. Colon cancer survival is associated with decreasing ratio of metastatic to examined lymph nodes. J Clin Oncol 2005; 23: 8706-8712
[31] Lee HY, Choi HJ, Park KJ et al. Prognostic significance of metastatic lymph node ratio in node-positive colon carcinoma. Ann Surg Oncol 2007; 14:1712-1717

[32] Rosenberg R, Friederichs J, Schuster T et al. Prognosis of patients with colorectal cancer is associated with lymph node ratio: a single-center analysis of 3,026 patients over a 25-year time period. Ann Surg 2008; 248: 968-978

[33] Derwinger K, Carlsson G, Gustavsson B. A study of lymph node ratio as a prognostic marker in colon cancer. Eur J Surg Oncol 2008; 34: 771-775

[34] Wang J, Hassett JM, Dayton MT et al. Lymph node ratio: role in the staging of node-positive colon cancer. Ann Surg Oncol 2008; 15: 1600-1608

[35] Schumacher P, Dineen S, Barnett $C$ et al. The metastatic lymph node ratio predicts survival in colon cancer. Am J Surg 2007; 194: 827-832

[36] Lykke J, Jess P, Roikjær O. A high lymph node yield in colon cancer is associated with age, tumour stage, tumour sub-site and priority of surgery. Results from a prospective national cohort study. Int J Colorectal Dis. 2016 Jul;31(7):1299-1305

[37] Telian SH, Bilchik AJ. Significance of the lymph node ratio in stage III colon cancer. Ann Surg Oncol 2008; 15: 1557-1558

[38] Sargent D, Sobrero A, Grothey A et al. Evidence for cure by adjuvant therapy in colon cancer: observations based on individual patient data from 20,898 patients on 18 randomized trials. J Clin Oncol 2009; 27: 872-877

[39] Petrelli F, Borgonovo K, Barni S. The emerging issue of ratio of metastatic to resected lymph nodes in gastrointestinal cancers: an overview of literature. Eur J Surg Oncol 2011; 37 : 836-847 